\title{
MRF Based Spatial Complexity for Hyperspectral Imagery Unmixing
}

\author{
Sen Jia and Yuntao Qian \\ College of Computer Science, Zhejiang University \\ Hangzhou 310027, P.R. China \\ zjujiasen@hotmail.com, ytqian@zju.edu.cn
}

\begin{abstract}
Hyperspectral imagery (HSI) unmixing is a process that decomposes pixel spectra into a collection of constituent spectra (endmembers) and their correspondent abundance fractions. Without knowing any knowledge of HSI data, the unmixing problem is transformed into a blind source separation (BSS) problem. Several methods have been proposed to deal with the problem, like independent component analysis (ICA). In this paper, we introduce spatial complexity that applies Markov random field (MRF) to characterize the spatial correlation information of abundance fractions. Compared to previous BSS techniques for HSI unmixing, the major advantage of our approach is that it totally considers HSI spatial structure. Additionally, a proof is given that spatial complexity is suitable for HSI unmixing. Encouraging results have been obtained in terms of unmixing accuracy, suggesting the effectiveness of our approach.
\end{abstract}

\section{Introduction}

Hyperspectral imagery (HSI) records data in hundreds of narrow contiguous spectral bands, which provides the opportunity to identify the ground materialsof-interest. Owing to the spatial resolution of the sensor, disparate materials may contribute to the spectrum measured from a single pixel, causing it a "mixed" pixel and making HSI unmixing a challenging problem in HSI applications. It is a process that decomposes pixel spectra into a collection of constituent spectra (endmembers) and their correspondent abundance fractions 112 .

At first, to make the problem simple, some methods unmix the HSI data under the circumstances that the knowledge of endmembers, including the spectral signatures and the number of endmembers, is known. And HSI unmixing is converted into a linear problem, which is easy to solve, such as spectral angle mapper [3]. But in most cases, the information of endmembers is not known, and the unmixing problem is transformed into a blind source separation (BSS) problem 4 . Several methods have been proposed to deal with the problem, like independent component analysis (ICA) [56]. However, the unmixing results of ICA are not satisfactory. That is, in any case, there are always endmembers incorrectly unmixed 7 .

Recently, a temporal complexity based BSS approach was proposed by Stone [8] which has shown success in separating linear mixtures of one-dimensional 
independent signals. It is to seek a weight vector that provides an orthogonal projection of mixtures such that each extracted signal is minimally complex. In this paper, for the sake of utilizing the spatial correlation information of abundance fractions, we extend the algorithm to two-dimensional spatial domain by Markov random field (MRF), named spatial complexity. Different from previous BSS techniques for HSI unmixing 9], our approach totally considers HSI spatial structure. In addition, we prove a theorem, which shows spatial complexity applicable for HSI unmixing.

The paper is organized as follows. First, the MRF based spatial complexity is presented. In Section 3, the corresponding gradient ascent algorithm is described; meanwhile, the pre- and post-processing are discussed. Section 4 presents experimental results of applying our approach to HSI data. Finally, some concluding remarks are given in Section 5 .

\section{Spatial Complexity Based HSI Unmixing}

HSI is a three-dimensional array with the width and length corresponding to spatial dimensions and the spectral bands as the third dimension, which are denoted by $I, J$ and $L$ in sequence. Let $\mathbf{R}$ be the image cube with each spectrum $\mathbf{R}_{i j}$ being an $L \times 1$ pixel vector where the boldface is used for vectors. Let $\mathbf{M}$ be an $L \times P$ spectral signature matrix that each column vector corresponds to an endmember spectrum and $P$ is the number of endmembers in the image. Let $\mathbf{S}$ be the abundance cube (the length of each dimension is $I, J$ and $P$ respectively) and every column $\mathbf{S}_{i j}$ be a $P \times 1$ abundance vector associated with $\mathbf{R}_{i j}$, with each element denoting the abundance fraction of relevant endmember present in $\mathbf{R}_{i j}$. Hence, the linear mixing model can be represented as follows:

$$
\mathbf{R}_{i j}=\mathbf{M S}_{i j}+\mathbf{n}
$$

where $\mathbf{n}$ is noise. The unmixing problem is to find a $P \times L$ matrix $\mathbf{W}$ for every pixel vector $\mathbf{R}_{i j}$

$$
\mathbf{Y}_{i j}=\mathbf{W R}_{i j}
$$

where $\mathbf{Y}$ is the estimated abundance cube of $\mathbf{S}$ and vector $\mathbf{Y}_{i j}$ expresses the fractional abundances of $P$ endmembers associated with $\mathbf{R}_{i j}$. Figure 1 gives the sketch map of HSI unmixing.

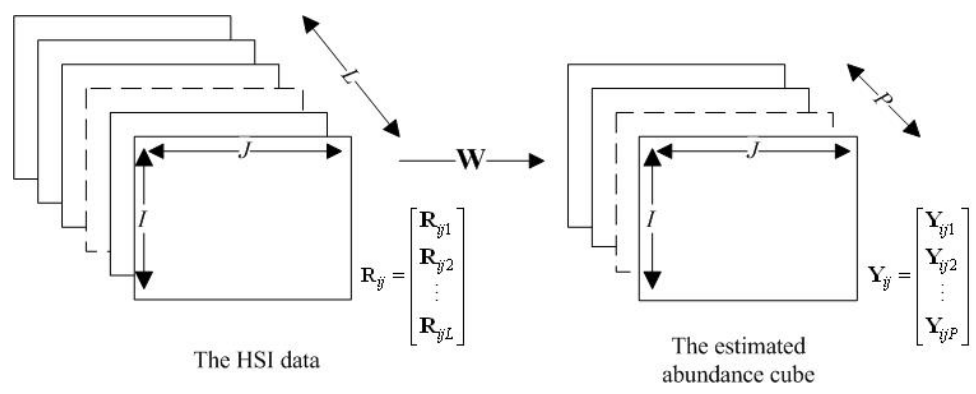

Fig. 1. The HSI unmixing sketch map 


\begin{tabular}{|c|c|c|c|c|}
\hline 5 & 4 & 3 & 4 & 5 \\
\hline 4 & 2 & 1 & 2 & 4 \\
\hline 3 & 1 & $\mathbf{Y}_{i j k}$ & 1 & 3 \\
\hline 4 & 2 & 1 & 2 & 4 \\
\hline 5 & 4 & 3 & 4 & 5 \\
\hline
\end{tabular}

(a)

\begin{tabular}{|c|c|c|}
\hline 0.05 & 0.2 & 0.05 \\
\hline 0.2 & & 0.2 \\
\hline 0.05 & 0.2 & 0.05 \\
\hline
\end{tabular}

(b)

Fig. 2. (a) The five order neighborhood system. (b) The weight matrix for $\lambda_{C}^{(t)}$.

In order to incorporate the spatial information of HSI data, we extend the method in [8], using spatial predictability to formulate complexity. The maximal predictability corresponds to minimal complexity, and vice versa. For $\mathbf{Y}$, its spatial predictability is defined as

$$
F(\mathbf{Y})=\sum_{k=1}^{P} \ln \frac{\sum_{i, j=1}^{I, J}\left(\overline{\mathbf{Y}}_{i j k}-\mathbf{Y}_{i j k}\right)^{2}}{\sum_{i, j=1}^{I, J}\left(\tilde{\mathbf{Y}}_{i j k}-\mathbf{Y}_{i j k}\right)^{2}}=\sum_{k=1}^{P} \ln \frac{V_{k}}{U_{k}}
$$

where $V_{k}$ and $U_{k}$ reflect the overall and local predictability degree of the $k$ th abundance image, respectively. Taking every pixel $\mathbf{Y}_{i j k}$ into account, $\overline{\mathbf{Y}}_{i j k}$ and $\tilde{\mathbf{Y}}_{i j k}$ reflect its overall and local spatial variability. Their definitions are

$$
\begin{aligned}
\overline{\mathbf{Y}}_{i j k} & =\frac{1}{I \times J-1} \sum_{t=1} \mathbf{Y}_{i j k}^{(t)} \\
\tilde{\mathbf{Y}}_{i j k} & =\sum_{t=1}^{N_{C}} \lambda_{C}^{(t)} \mathbf{Y}_{i j k}^{(t)}
\end{aligned}
$$

where $\mathbf{Y}_{i j k}^{(t)}, N_{C}$ and $\lambda_{C}^{(t)}$ are taken from the neighborhood system of MRF model.

MRF models are mainly used in feature extraction and image segmentation [10. For any pixel $\mathbf{Y}_{i j k}$ (for brevity's sake, $y$ is used to express $\mathbf{Y}_{i j k}$ ), its $n$ thorder neighborhood system is $N_{y}^{n}=\left\{y+\left.r\left|y+r \in N_{y},\right| r\right|^{2} \leq D[n]\right\}$, where $N_{y}$ are the neighbors of $\mathbf{Y}_{i j k}$ (the adjacent pixels except itself; for details, see [10]), $|r|$ denotes the Euclidian distance between sites $y$ and $y+r$, and $D[n]$ is a member of the set of all possible integers defined as $D=\left\{D[n] \mid D[n]=p^{2}+q^{2}, p, q \in\right.$ $\mathbb{Z}, D[k]>D[l]$ if $k>l\}$. Figure 2(a) displays the five order neighborhood system. It labels first-order spatial neighbors of site $\mathbf{Y}_{i j k}$ as "1", second-order neighbors as "2", and so on. Concerning above three parameters, $\mathbf{Y}_{i j k}^{(t)}$ denotes the $t$ thorder neighbors of $\mathbf{Y}_{i j k}, N_{C}$ is set to 2 for $\tilde{\mathbf{Y}}_{i j k}$, and the corresponding weight matrix of $\lambda_{C}^{(t)}$ is shown in Figure 2(b). Here, $\overline{\mathbf{Y}}_{i j k}$ and $\tilde{\mathbf{Y}}_{i j k}$ can be regarded as the energy functions of $\mathbf{Y}_{i j k}$ in different range.

However, some conditions must be satisfied for spatial complexity. That is, the number of mixtures is much greater than that of sources, which can be derived from the following theorem.

Theorem 1. As the number of mixtures increases relative to a fixed number of sources, the difference between the extreme values (both minimum and 
maximum) of mixtures' and sources' spatial complexity decreases, which offers more possibilities to extract all sources.

The proof of the theorem is in the appendix. It explains why the sources could not be totally separated out when the number of mixtures is close to that of sources, leading to the unsuitability of spatial complexity for multispectral imagery (MSI, only containing several spectral bands). For HSI data, because the number of bands (mixtures number) is much larger than that of endmembers (sources number, $L \gg P$ ), spatial complexity is a suitable approach for HSI unmixing. In addition, it should be noted that the correlation among neighboring abundance fractions of endmember guarantees its validity.

\section{The Gradient Ascent Algorithm and Pre- \& Post-processing}

To extract the abundance cube in parallel, gradient ascent algorithm is employed. Given $\mathbf{W}_{k}$ being a $1 \times L$ row vector of $\mathbf{W}$, equation (3) can be rewritten as

$$
F(\mathbf{Y})=F(\mathbf{W})=\sum_{k=1}^{P} \ln \frac{\mathbf{W}_{k} \overline{\mathbf{C}} \mathbf{W}_{k}^{\mathrm{T}}}{\mathbf{W}_{k} \tilde{\mathbf{C}} \mathbf{W}_{k}^{\mathrm{T}}}
$$

where $\overline{\mathbf{C}}$ and $\tilde{\mathbf{C}}$ are both $L \times L$ matrixes of overall and local covariances between mixtures respectively. They can be defined as

$$
\begin{aligned}
& \overline{\mathbf{C}}=\sum_{i, j=1}^{I, J}\left(\overline{\mathbf{R}}_{i j}-\mathbf{R}_{i j}\right)\left(\overline{\mathbf{R}}_{i j}-\mathbf{R}_{i j}\right)^{\mathrm{T}} \\
& \tilde{\mathbf{C}}=\sum_{i, j=1}^{I, J}\left(\tilde{\mathbf{R}}_{i j}-\mathbf{R}_{i j}\right)\left(\tilde{\mathbf{R}}_{i j}-\mathbf{R}_{i j}\right)^{\mathrm{T}}
\end{aligned}
$$

$\overline{\mathbf{C}}$ and $\tilde{\mathbf{C}}$ only need to be computed once, which greatly alleviate the computational load. $\overline{\mathbf{R}}_{i j}=\left[\overline{\mathbf{R}}_{i j 1}, \overline{\mathbf{R}}_{i j 2}, \ldots, \overline{\mathbf{R}}_{i j L}\right]^{\mathrm{T}}, \tilde{\mathbf{R}}_{i j}=\left[\tilde{\mathbf{R}}_{i j 1}, \tilde{\mathbf{R}}_{i j 2}, \ldots, \tilde{\mathbf{R}}_{i j L}\right]^{\mathrm{T}}$, and the definitions of $\overline{\mathbf{R}}_{i j k}$ and $\tilde{\mathbf{R}}_{i j k}$ are similar to equation (4). The derivative of $F$ with respect to $\mathbf{W}$ is

$$
\nabla_{\mathbf{W}} F=2 \mathbf{W} \overline{\mathbf{C}} . / \mathbf{V}-2 \mathbf{W} \tilde{\mathbf{C}} . / \mathbf{U}
$$

The sign ./ means "point division", namely, each element of numerator matrix divides the corresponding element of denominator matrix. $\mathbf{V}$ is a $P \times L$ matrix with the $k$ th row vector acquired by replicating $V_{k}$. Likewise, the $k$ th row vector of $\mathbf{U}$ is the replication of $U_{k}$. The gradient ascent rule is

$$
\mathbf{W}_{\text {new }}=\mathbf{W}_{\text {old }}+\eta \nabla_{\mathbf{W}} F
$$

where $\eta$ is the learning rate. To speed up the convergence of $\mathbf{W}$, a scheme is used on $\eta$. In the experiments conducted in Section 4, 0.1 is assigned as its initial value, then 0.9 is multiplied every other twenty steps.

Some methods should be applied to process the HSI data before and after the unmixing algorithm to make the results more accurate. Preprocessing (centering and whitening) through principle component analysis (PCA) 4] is adopted as a means to reduce the relevance of first and second statistics, and to speed 
up the convergence process. Postprocessing step is to identify the position of the endmembers, and the method in [1112 is employed. Briefly speaking, the histogram and the skewness of every abundance image are computed first, and the sign of skewness indicates the direction to be searched for the threshold (Positive to the right of the center, otherwise to the left). Then the pixel value where the histogram firstly takes zero is selected as the threshold. Finally, the threshold is used to filter corresponding abundance image. Readers are referred to [11] for details.

At last, the pseudo-code of spatial complexity for HSI unmixing is specified.

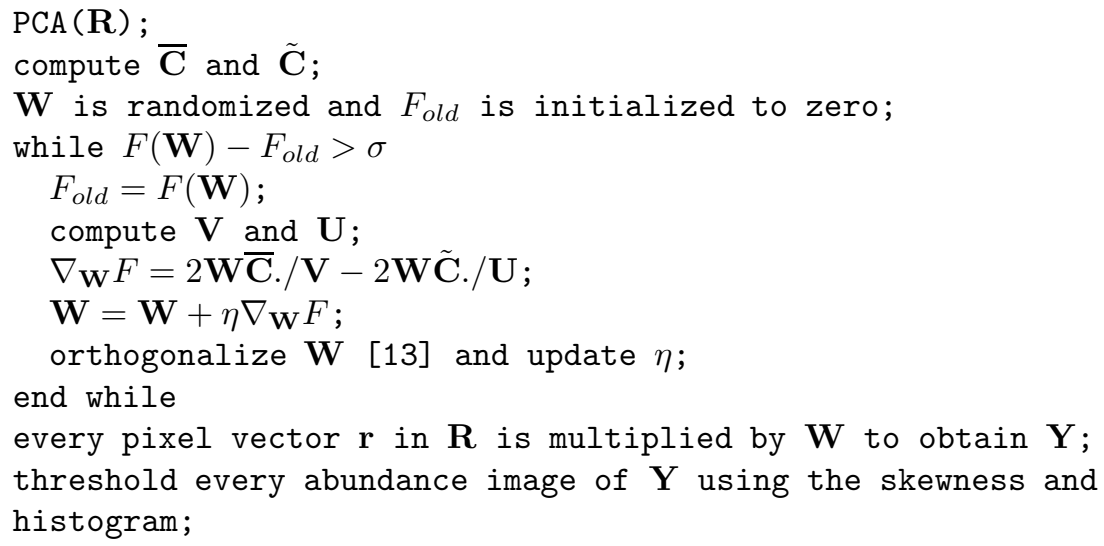

\section{Experimental Results}

In this section, two sets of real hyperspectral image data, HYDICE (HYperspectral Digital Imagery Collection Experiment) [14 and PHI (Pushbroom Hyperspectral technique Imager) are applied to evaluate the performance of spatial complexity. Some methods have been proposed to estimate the number of endmembers $\mathrm{P}$, such as virtual dimensionality [15. In this paper, we assume that it is known in advance to maximize the efficiency of spatial complexity. In addition, the unmixing results of undercomplete ICA (UICA) algorithm [16] are given for comparative analysis.

\subsection{HYDICE Data}

Figure 3 shows an urban scene of size $307 \times 307$ extracted from HYDICE data. It is composed of 210 spectral channels with spectral resolution $10 \mathrm{~nm}$ acquired in the $400 \mathrm{~nm}$ and 2.5 micron region. After low signal-to-noise ratio (SNR) bands are removed, only 166 bands remain (i.e., $L=166$ ). According to the ground truth, this data contain four materials: vegetation, asphalt, soil and roof. So $P$ is set to 4 .

Firstly, the UICA algorithm is applied to the dataset. Figure 4 presents the unmixing results. Except that the asphalt and soil are classified in Figure 4(b) and 4(c) the other two images are mixtures, which verify the conclusion of [7]: In any case, there are always endmembers incorrectly unmixed. Then spatial 


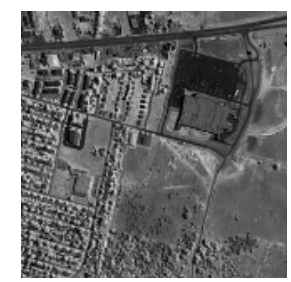

Fig. 3. The urban scene extracted from HYDICE HSI

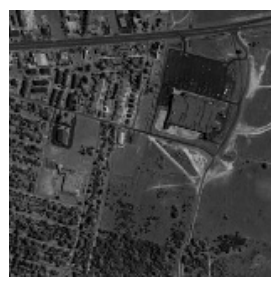

(a) mixture

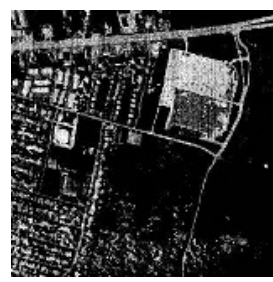

(b) asphalt

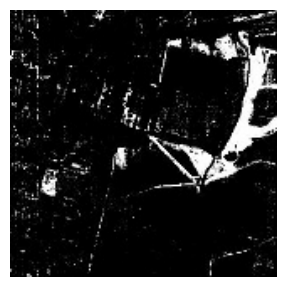

(c) soil

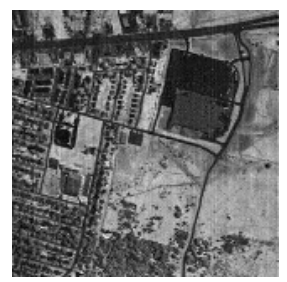

(d) mixture

Fig. 4. Unmixing results produced by UICA

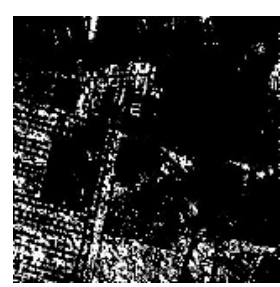

(a) vegetation

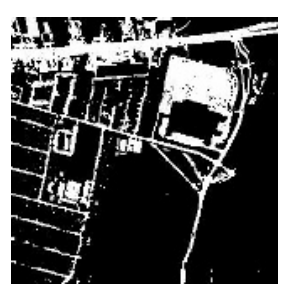

(b) asphalt

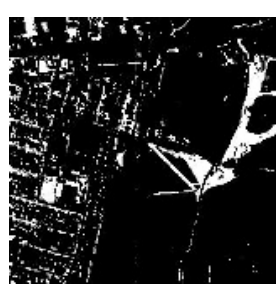

(c) soil

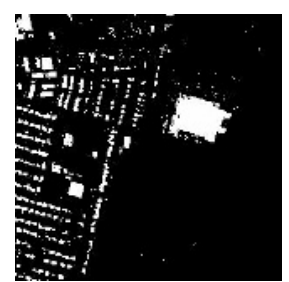

(d) roof

Fig. 5. Unmixing results produced by spatial complexity

complexity is utilized, and the results are displayed in Figure 5. Different from Figure 4, all the four materials: vegetation, asphalt, soil and roof are successfully extracted. For convenience, we let white stand for the separated materials and black for the background in the unmixing results. Comparing the abundance distribution of asphalt and soil which are both unmixed by the two methods, it is clear that the results of spatial complexity are much better than those of UICA. Concretely speaking, Figure 4(b) misclassifies some vegetation pixels as asphalt, and 4(c) only classifies large pieces of soil but ignores small ones. Contrarily, Figure 5(b) and 5(c) totally extract the two materials. It is worth noting that the unmixing results in Figure 5 have been filtered by the postprocessing method, so they are more distinct. Hence, we can conclude that spatial complexity is more efficient than UICA.

\subsection{PHI Data}

The PHI data used in the following experiment were directly extracted from the PHI image scene of size $400 \times 331$ shown in Figure 6. The image data were ac- 


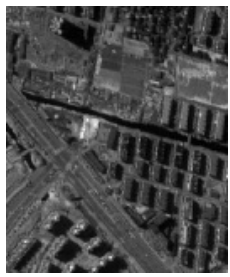

Fig. 6. The subscene of Shanghai World Expo Garden extracted from PHI HSI

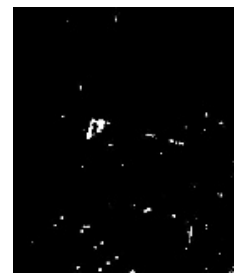

(a) water

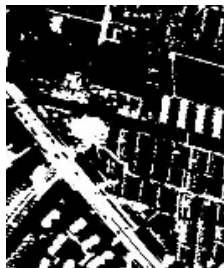

(b) road

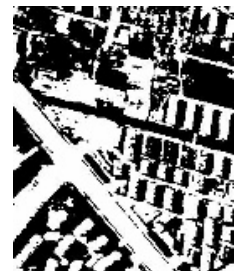

(c) mixture

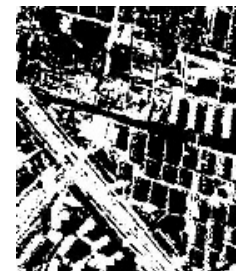

(d) mixture

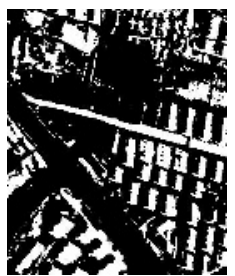

(e) mixture

Fig. 7. Unmixing results produced by UICA

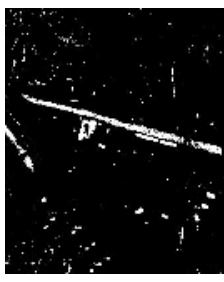

(a) water

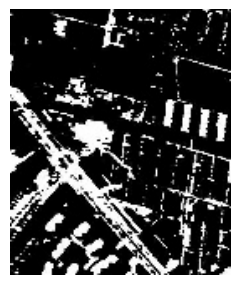

(b) road

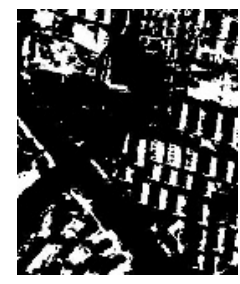

(c) roof

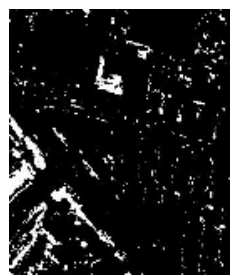

(d) soil

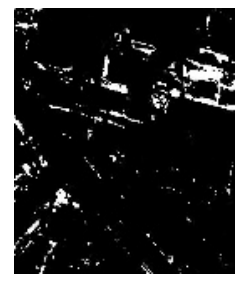

(e) sparse grass

Fig. 8. Unmixing results produced by spatial complexity

quired from the subscene of Shanghai World Expo Garden in October 2003 with the ground sampling distance approximately $1.2 \mathrm{~m}$. It has 124 spectral channels ranging from $400 \mathrm{~nm}$ to $990 \mathrm{~nm}$ with spectral resolution $5 \mathrm{~nm}$. And no spectral bands are removed in the experiments (i.e., $L=124$ ). According to the ground truth, this data contain five materials: water, road, roof, soil and sparse grass. So $P$ is assigned to 5 .

Same as the above experiment, the UICA algorithm is firstly applied. Figure [7] shows the unmixing results. Except that the water and road are extracted out in Figure 7(a) and $7(\mathrm{~b})$, all the other three images are mixtures, which verify the conclusion of [7] again. The unmixing results generated by spatial complexity are illustrated in Figure 8. All the five materials: water, road, roof, soil and sparse grass, are displayed in sequence. Comparing images (a) and (b) between Figure 7 and 8 , it is easy to find that the abundance of water extracted in Figure 7(a) is just a small part of Figure 8(a). So UICA actually separated out only one material: road. And the same conclusion as the above subsection can be drawn. 


\section{$5 \quad$ Summary and Conclusions}

We have presented a spatial complexity based algorithm for hyperspectral imagery unmixing. The algorithm extends the temporal complexity; the major improvement is the better representation of spatial correlation of abundance image. A proof is presented that spatial complexity is suitable for HSI unmixing. Its effectiveness has been tested by comparison to UICA with data from $\mathrm{HY}$ DICE and PHI HSI. The experimental results show that our approach provides a promising method for HSI unmixing.

The reason that ICA could not totally separate out the sources (the abundance cube) ascribes to the dependence among the abundance fractions (i.e., the sum of them associated to each pixel is constant due to physical constraints in the data acquisition process) [7]. Spatial complexity is actually a second order statistics algorithm, which does not use explicitly or implicitly any criterion of statistical independence [4. Hence, it is reasonable to introduce the method to unmix HSI data, and the experimental results confirm it. However, the conjecture in [8] is proved based on sources independence; so in future work, we will attempt to find out the connection between spatial complexity and sources independence.

\section{Acknowledgment}

The authors would like to thank Shanghai Institute of Technical Physics, Chinese Academy of Sciences for providing the PHI data.

\section{References}

1. D. Manolakis and G. Shaw. Detection algorithms for hyperspectral imaging applications. IEEE Signal Processing Magazine, 19(1):29-43, January 2002.

2. N. Keshava. A survey of spectral unmixing algorithms. Lincoln Lab Journal, 14(1):55-73, 2003.

3. R.H. Yuhas, A.F.H. Goetz, and J.W. Boardman. Discrimination among semiarid landscape endmembers using the spectral angle mapper (sam) algorithm. In Summaries of the 3rd Annual JPL Airborne Geoscience Workshop, volume 1, pages 147-149, 1992.

4. A. Cichocki and S. Amari. Adaptive Blind Signal and Image Processing: Learning Algorithms and Applications. John Wiley \& Sons, 2002.

5. J.D. Bayliss, J.A. Gualtieri, and R.F. Cromp. Analyzing hyperspectral data with independent component analysis. In Proceeding of SPIE Applied Image and Pattern Recognition Workshop, volume 3240, pages 133-143, 1997.

6. S.-S. Chiang, C.-I. Chang, J.A. Smith, and I.W. Ginsberg. Linear spectral random mixture analysis for hyperspectral imagery. IEEE Transaction on Geoscience and Remote Sensing, 40(2):375-392, February 2002.

7. J.M.P. Nascimento and J.M.B. Dias. Does independent component analysis play a role in unmixing hyperspectral data? IEEE Transaction on Geoscience and Remote Sensing, 43(1):175-187, January 2005.

8. J.V. Stone. Independent Component Analysis: A Tutorial Introduction. MIT Press, 2004 . 
9. Q. Du and S. Chakrarvarty. Unsupervised hyperspectral image classification using blind source separation. In IEEE International Conference on Acoustics, Speech, and Signal Processing 2003, volume 3, pages 437-440, 2003.

10. H. Deng and D.A. Clausi. Unsupervised image segmentation using a simple mrf model with a new implementation scheme. Pattern Recognition, 37(12):2323-2335, 2004.

11. S.-S. Chiang, C.-I. Chang, and I.W. Ginsberg. Unsupervised target detection in hyperspectral images using projection pursuit. IEEE Transaction on Geoscience and Remote Sensing, 39(7):1380-1391, July 2001.

12. S.A. Robila and P.K. Varshney. Target detection in hyperspectral images based on independ-ent component analysis. In Proceeding of SPIE Automatic Target Recognition, volume 4726, pages 173-182, 2002.

13. A. Hyvärinen. Fast and robust fixed-point algorithms for independent component analysis. IEEE Transactions on Neural Networks, 10(3):626-634, 1999.

14. The HYDICE HSI dataset. http:// www.tec.army.mil/Hypercube/.

15. C.-I Chang and Q. Du. Estimation of number of spectrally distinct signal sources in hyperspectral imagery. IEEE Transaction on Geoscience and Remote Sensing, 42(3):608-619, March 2004.

16. J.V. Stone and J. Porrill. Undercomplete independent component analysis for signal separation and dimension reduction. Technical report, Psychology Department, Sheffield University, http:// www.shef.ac.uk/ pc1jvs/, 1998.

17. S. Xie, Z. He, and Y. Fu. A note on stone's conjecture of blind signal separation. Neural Computation, 17(2):321-330, February 2005.

\section{Appendix: Proof of Theorem 1}

The conjecture used in [8] has been modified and proved by Xie et al. [17]. Namely, any mixture has a complexity lying between its least and most complex source signals. Although it aims at one-dimensional signal, it also holds for twodimensional image (The proof is similar to Xie's, so it is not given), which can be described that the spatial complexity of every mixture lies between its least and most complex sources.

Let $C(\mathrm{X})$ denote the spatial complexity of $\mathrm{X}, \mathrm{X} \uparrow$ denote the augment of $\mathrm{X}$, $\mathrm{X} \rightarrow \mathrm{Y}$ denote that $\mathrm{X}$ approaches to $\mathrm{Y}$, and $\mathrm{X} \Rightarrow \mathrm{Y}$ denote that $\mathrm{X}$ implies $\mathrm{Y}$. First, the Xie's conjecture can be formalized as

$$
\min (C \text { (sources })) \leq C \text { (mixtures }) \leq \max (C \text { (sources }))
$$

Equation (2) indicates that the estimated sources are the "mixing" of mixtures. According to the Xie's conjecture, it is clear that

$$
\min (C \text { (mixtures })) \leq C \text { (estimated sources }) \leq \max (C \text { (mixtures }))
$$

Second, without loss of generality, due to the randomness of the mixing process [6], the following formulas can be obtained

$$
\begin{aligned}
& \min (C \text { (mixtures })) \rightarrow \min (C \text { (sources })) \\
& \text { mixture number } \uparrow \\
& \max (C \text { (mixtures })) \rightarrow \max (C \text { (sources })) \\
& \text { mixture number } \uparrow
\end{aligned}
$$


which means

$$
\begin{aligned}
& R \text { (mixtures) } \uparrow \rightarrow R(\text { sources }) \\
& \text { mixture number } \uparrow
\end{aligned}
$$

where $R(\mathrm{X})$ is defined as

$$
R(\mathrm{X})=\max (C(\mathrm{X}))-\min (C(\mathrm{X}))
$$

Correspondingly, from (10),

$$
\begin{gathered}
R \text { (mixtures) } \uparrow \\
\text { mixture number } \uparrow
\end{gathered}
$$

Considering (12) and (14) simultaneously,

$$
\begin{aligned}
& R \text { (estimated sources) }) \rightarrow R(\text { sources }) \\
& \quad \text { mixture number } \uparrow
\end{aligned}
$$

which can derive the following formula

$$
\begin{aligned}
& \text { (estimated sources) } \\
& \text { mixture number } \uparrow
\end{aligned}
$$

Consequently, the conclusion can be drawn. 